\title{
Zolpidem dependence with frontal lobe syndrome: a case report
}

\author{
Udayan Majumder $^{1 *}$, Rajesh Das ${ }^{2}$, Rajkumar Lenin Singh ${ }^{1}$
}

${ }^{1}$ Department of Psychiatry,

${ }^{2}$ Department of Pharmacology, Regional Institute of Medical Sciences, Imphal, Manipur, India

Received: 25 September 2017 Accepted: 27 October 2017

*Correspondence to:

Dr. Udayan Majumder, Email: udayan586@gmail.com

Copyright: (C) the author(s), publisher and licensee Medip Academy. This is an openaccess article distributed under the terms of the Creative Commons Attribution NonCommercial License, which permits unrestricted noncommercial use, distribution, and reproduction in any medium, provided the original work is properly cited.

\begin{abstract}
Zolpidem is a non-benzodiazepine sedative hypnotic that binds to the benzodiazepine binding site on the gammaaminobutyric acid type A (GABA-A) receptors. It is the most commonly prescribed sleep medication which has been shown to be effective for treating insomnia on a short-term basis with fewer side effects than traditional benzodiazepines, which are feared for their abuse and dependence potential. Many studies have reported efficacy and safety of zolpidem in treatment of insomnia keeping in mind about its low abuse, and dependence capability. We present a case of zolpidem dependence in a 36-yearold male to emphasize that zolpidem also should be judiciously prescribed under supervision so that it does not develop tolerance, abuse and dependence.
\end{abstract}

Keywords: Dependence, Frontal lobe syndrome, Insomnia, Zolpidem

\section{INTRODUCTION}

Insomnia disorder is the most prevalent sleep disorder, diagnosed when there is difficulty getting to sleep and/or staying asleep, with distress regarding sleep issue and/or daytime impairment. Zolpidem is the most commonly prescribed sleep medication, where almost 39 million prescriptions written in the United States in 2011.,2 However, in contrast to BDZs, zolpidem shows selectivity for the V1 receptor subtype, which corresponds to GABA A receptors containing the a1 subunit 1 . Zolpidem is able to produce sedation without interfering with the BDZ properties linked to other receptor subtypes. ${ }^{3}$ So zolpidem was considered a safer hypnotic than benzodiazepines because of a lesser liability for abuse and dependence.
However, in recent years, new evidence has revealed that the behavioral effects of zolpidem at higher than recommended doses are generally similar to those of BDZs. As the use of benzodiazepines (BDZs) in the treatment of insomnia has been declining in recent years as a result of studies documenting a series of deleterious effects (addiction, rebound insomnia, etc.), the prescription of non-BDZ hypnotics, such as zolpidem has been increasing substantially. There is some indication that zolpidem can have an anxiolytic effect at higher doses and that with increasing dose of zolpidem for sleep can lead to withdrawal side effects during the daytime. ${ }^{4}$ The guideline also states that the duration of treatment should usually vary from a few days to two weeks with a maximum of four weeks, including tapering off where appropriate. 5 However, recently concern has been raised regarding the dependence potential of these Z-drugs. ${ }^{6}$ In the last few 
years, several cases of misuse of the Z-drugs characterized by significant dose escalation, dependence, abuse and withdrawal symptoms have been reported. ${ }^{7-10} \mathrm{We}$ present a case of zolpidem dependence in a 36-year-old male to highlight the need for caution when prescribing this drug.

\section{CASE REPORT}

The patient 32 years old married male from urban setting, teacher by profession presented to the psychiatry outpatient facility of our institute with sleep disturbances and inability to stop zolpidem use. He started to use spasmo-proxyvon (dextropropoxyphene) tablets recreationally since 2011 while after about 1 year he started abusing the same with a daily intake of 16 tablets per day. Nearly one and a half year back in 2015 one day he consumed a relatively higher dose of tablets than usual (nearly 30 tablets) and had a fainting attack followed by head injury with extradural hemorrhage which required prompt neurosurgical intervention.

During discharge he was prescribed tablet zolpidem 10mg for insomnia. Despite using usual dose of Zolpidem he couldn't initiate sleep at night and could sleep for a maximum duration of 3-4 hours with frequent awakenings. Hence he started self medication and increased taking 2-3 tablets of zolpidem. Following that he could sleep better and had a sense of euphoria. He enjoyed the high and gradually started increasing the dose to 2-3 tablets every 7-10 days and during presentation he was taking 24-26 tablets (240-260mg) of Zolpidem 10mg in 4 divided doses over 24 hours. Whenever he didn't consume zolpidem he developed insomnia, irritability, generalized weakness, excessive desire to take the same every after 4-6 hours which went on increasing day by day.

Despite knowing the harmful effects of consuming unsupervised higher dose of the drug he couldn't stop himself from taking it. His family, socio-occupational and cultural life was impaired to great extent and gradually he spent most of his time in procuring the drug from pharmacies. Since last 15-18 months he also developed persistent low mood present almost whole day for most of the days, generalized lack of interest in pleasurable activities and his teaching job, easy fatigability, lack of motivation and dizziness off and on.

He was assessed and admitted in psychiatric ward and detoxification was started with Diazepam 20mg tablet with Clobazam 10mg tablet in divided doses. Gradually the benzodiazepines were tapered off over 15 days. 6 sessions of motivational enhancement therapy were provided along with relapse prevention. After detoxification he was educated about sleep hygiene measures and the need to abstain from benzodiazepines and other hypnotics. He has till date been on follow-up for more them 2 months. He is having normal sleep and is abstinent from zolpidem and other hypnotics.

\section{DISCUSSION}

Zolpidem is a non-benzodiazepine hypnotic. Its mechanism of action is selective benzodiazepine type 1 receptor agonist. However, other studies suggest at higher does it loses selectivity and may have addictive potential similar to benzodiazepines. ${ }^{10}$ Rappa et al described a case of zolpidem dependence detoxified using a 7 days tapering regime of diazepam. Our patient was als o treated with a 15 weeks tapering dose of diazepam and clobazam. It has been proposed that possible GABA-A receptor mutations may be a predisposing factor in zolpidem dependency. ${ }^{11}$ Literature suggests that patients with a history of drug abuse or dependence or those with psychiatric disorders are at increased risk of abuse of zolpidem and other Zdrugs, which is also consistent with our study. ${ }^{12}$

In view of several case reports of Z-drugs dependence in the last few years, it is suggested that the same stringent precautions as with benzodiazepines should be adhered to while prescribing these Z-drugs. Since there are presently no clear-cut guidelines for the treatment of Z-drugs dependence, prevention is probably the best form of treatment. There is also an urgent need to study the range of treatment options for dependent patients, but it would be expected that gradual withdrawal would be as prominent as with benzodiazepines.

Funding: No funding sources

Conflict of interest: None declared

Ethical approval: Not required

\section{REFERENCES}

1. Ohayon MM. Epidemiology of insomnia: what we know and what we still need to learn. Sleep Med Rev. 2002;6(2):97-111.

2. IMS, Vector One: National (VONA) and Total Patient Tracker (TPT). Year 2011. Extracted June 2012.

3. Mitler MM. Nonselective and selective benzodiazepine receptor agonists where are we today? Sleep. 2000;23(1):39-47.

4. Rush CR, Baker RW, Wright K. Acute behavioral effects and abuse potential of trazodone, zolpidem and triazolam in humans. Psychopharmacology. 1999; 144(3):220-33.

5. National Institute for Clinical Excellence (NICE). Guidance on the use of zaleplon, zolpidem and zopiclone for short term management of insomnia. London: NICE (Technology Appraisal 77); 2004.

6. Damm J, Eser D, Moeller HJ, Rupprecht R. Severe dependency on zolpidem in a patient with multiple sclerosis suffering from paraspasticity. World $\mathrm{J}$ Biol Psychiatry. 2010;11(2):516-8.

7. Sethi PK, Khandelwal DC. Zolpidem at supratherapeutic doses can cause drug abuse, dependence and withdrawal seizures. J Assoc Physicians India. 2005;53:139-40.

8. Pitchot W, Ansseau M. Zolpidem dependence and withdrawal seizure. Rev Med Liege. 2009;64:407-8. 
9. Wang LJ, Ree SC, Chu CL, Juang YY. Zolpidem dependence and withdrawal seizure report of two cases. Psychiatr Danub. 2011;23(1):76-8.

10. Rappa LR, Larose-Pierre M, Payne DR, Eraikhuemen NE, Lanes DM, Kearson ML. Detoxification from High-dose zolpidem using Diazepam. Ann Pharmacother. 2004;38:590-4.

11. Liappas IA, Malitas PN, Dimopoulos NP, Gitsa OE, Liappas AI, Nikolaou ChK, et al. Zolpidem dependence case series: possible neurobiological mechanisms and clinical management. J Psychopharmacol. 2003; 17(1):131-5.
12. Hajak G, Muller WE, Witchen HU, Pittrow D, Kirch W. Abuse and dependence potential for the nonbenzodiazepine hypnotics zolpidem and zopiclone: a review of case reports and epidemiological data. Addiction. 2003;98(10):1371-8.

Cite this article as: Majumder U, Das R, Singh RKL. Zolpidem dependence with frontal lobe syndrome: a case report. Int J Basic Clin Pharmacol 2017;6:2936-8. 